\title{
Medical Ethics Knowledge and Practice among Healthcare Workers at Prince Mansour Military Hospital (PMMH), Taif, Saudi Arabia, 2012
}

\author{
Muied A. AL Qarny ${ }^{1 *}$, Amal M. Alsofiani ${ }^{2}$, Ali Githan AL Garny ${ }^{3}$ \\ ${ }^{1 *}$ Family Medicine Department, Ministry of National Guard, \\ 2Family Medicine Department, Ministry of Health, \\ ${ }^{3}$ Family Medicine Department, Prince Mansour Military Hospittal, Taif, Saudi Arabia.
}

\begin{abstract}
Background: Medical ethics require the physician to do what is best for the patient and place the patient's interests before the interests of the physician. It is not about avoiding harm; rather, it is a set of norms, values and principles.

Objectives: To assess medical ethics knowledge and practice and its associated factors among healthcare workers at PMMH, Taif, Saudi Arabia, 2012.

Methodology: This was a cross sectional study including all healthcare workers at PMMH who were present at the time of study conduction. Self-administered questionnaire which had been validated in previous published studies was utilized for data collection. The questionnaire is composed of 16 points which cover the 6 of the values that commonly apply to medical ethics as followings: autonomy, beneficence, non-maleficence, justice, dignity and truthfulness and honesty. Participates were assessed for knowledge regarding each single aspect of ethical issues with a grade scale (likert scale) from 1 to 3 (ranging from disagree to agree).
\end{abstract}

Results: Overall, 224 of 270 health care workers, invited to participate in the study by filling out the research questionnaire, returned completed questionnaires, giving a response rate of $83.0 \%$. Their age ranged between 24 and 52 years with a mean of 31.7 (SD 6.0) years. Three-quarters of them (168; $75.0 \%$ ) were females. $42.4 \%$ of healthcare workers had bad knowledge regarding medical ethics, mostly doctors $(56.8 \%$

\section{INTRODUCTION}

The global medical profession has maintained simple ethical standards for more than 4,000 years. For example, the Hippocratic Oath, the Holy Quran, the field is indebted to Muslim medicine such as Ishaq bin Ali Rahawi (who wrote the Conduct of Physician, the first book dedicated to medical ethics) and Muhammad ibnZakariyaar-Razi (known as Rhazes in the West), as well as cultures, traditions, and social morality have shaped and guided the development of ethical standards in the medical profession. The majority of these historical documents focus on "avoiding harm to patients".

Medical ethics is a relatively new subspecialty in the medical field and is still in its infancy in many parts of the world. Healthcare ethics is not routinely taught to the medical professionals, and there are reports that even the word "ethics" has been completely ignored during the undergraduate medical curriculum .The same versus $35.3 \%$ of nurses). Very good or excellent level of medical ethics knowledge was reported among $21.4 \%$ and $7.1 \%$ of healthcare workers, respectively. Nurses reported more significant very good and excellent knowledge than doctors (30\% versus $25.7 \%$ ).

Conclusion: More than $40 \%$ of healthcare workers in $\mathrm{PMMH}$ had bad knowledge regarding medical ethics, mostly doctors. Nurses reported more significant medical ethics knowledge than doctors. There were varying opinions in many issues of care-ethics.

Keywords: Doctors, Ethics, Healthcare, Likert scale, Nurses.

\section{*Correspondence to:}

Dr. Muied A. AL Qarny

Assistant Consultant of Family Medicine, Ministry of National Guard, Taif, Saudi Arabia.

\section{Article History:}

Received: 23-07-2016, Revised: 27-07-2016, Accepted: 31-07-2016

\begin{tabular}{|l|c|}
\hline \multicolumn{2}{|c|}{ Access this article online } \\
\hline Website: & Quick Response code \\
www.jimrp.com & \\
\hline DOI: & \\
10.21276/ijmrp.2016.2.4.030 & \\
\hline
\end{tabular}

may be true with the training of other healthcare providers such as the nursing professionals. Hence it is not surprising that the theory and application of healthcare ethics in day-to-day practice are still not well known to many healthcare providers. In such a situation, practice of ethics in healthcare will be very much influenced by the cultural background and beliefs of the people in every region. ${ }^{2}$

Majority of the literature and teaching on healthcare ethics emphasize on the so called "western" dimensions of ethics which are formulated and applicable to the healthcare profession in the western countries. However, there have been many recommendations that ethics in medicine has to be formulated within the context of the individual socio-economic, geo-political, religious and cultural background of a particular region. ${ }^{2}$

Traditional medical training offers little help in resolving the ethical dilemmas encountered by healthcare professionals. Although 
teaching of medical ethics was introduced into the medical curriculum of the Faculty of Medical Sciences and medical faculty in most of Saudi Arabia Universities recently, it has been didactic in a lecture theatre setting and taught as relevant to the various branches of medicine and not as a separate course to be evaluated in its own merit. Even among other healthcare professionals there has been no effort to teach ethics as separate courses incorporated into the curriculum. My hypothesis is that there is a lack of knowledge that reflected in the healthcare workers attitude in dealing with ethical issues. ${ }^{2}$

Traditional medical oaths and codes prescribe a physician's character, motives, and duties. Typically they portray ideal physicians as devoted to the welfare of patients and to advancement of the medical profession and medical knowledge, responding compassionately to the suffering of patients, humbly mindful of the limits of their curative powers and the harms they may unintentionally cause. The Hippocratic injunction "strive to help, but above all, do no harm" is the ruling maxim. In current discussion, this maxim has been codified in oft-cited "principles of non-maleficence and beneficence". ${ }^{3}$ The present study aimed to assess medical ethics knowledge and practice and their determinants among healthcare workers at PMMH, Taif, Saudi Arabia, 2012.

\section{SUBJECTS AND METHODS}

A cross sectional study was conducted among healthcare workers at Prince Mansour Military hospital PMMH),Taif, Saudi Arabia. Taif is a city in Makkah Al-Mokarramah Province of Saudi Arabia at an elevation of $1,879 \mathrm{~m}(6,165 \mathrm{ft}$.) on the slopes of the Sarawat Mountains (Al-Sarawat Mountains). It has a population of 1,011,613 (2010 census). In Taif, there are around 5 governmental hospitals and 114 primary health care centres. The study was conducted at PMMH (36 beds) which is a component of the directorate of Armed Forces Hospital in Taif. Pharmacists, social workers, radiographers, laboratory technicians, support staff such as porters and maids and clerical staff and administration staff were excluded. All healthcare workers at PMMH who were present at the time of study conduction were invited to participate in the study. According to the data obtained from the medical directorate of PMMH, the total number is 326 (137 physicians and 189 nurses). Thirty eight were outside the kingdom and 18 were at rotations during conduction of the study. Thus, 270 health care workers (102 physicians and 168 nurses) were invited to participate in the study.

Self-administered questionnaire which had been validated in previous published studies, ${ }^{2}$ the questionnaire is composed of 16 points, compared to the original questionnaire of 10 items. However, due to differences in our culture, social and religious values, those 6 points, were added. The questionnaire was validated by 3 consultant Family \& community medicine (face validity). Moreover, these 6 points were mentioned in other studies. The initial part of the questionnaire consisted of demographics such as occupation, age, gender, nationality and duration of the respondents' work experience in their practice. Permission was taken to use the questionnaire. The questionnaire consists of 16 points, which cover the 6 of the values that commonly apply to medical ethics as followings: autonomy, beneficence, non-maleficence, justice, dignity, truthfulness and honesty.
It was designed to examine the respondent's decision-making process in relation to ethical and legal problems and to identify the healthcare workers knowledge, practice. In each point, we try to assess participates knowledge regarding each single aspect of ethical issues with a grade scale (likert scale) from 1 to 3 (ranging from disagree to agree).

A permission from Joint Program of Family Medicine to conduct the research has been obtained as well as individual consents were filled by participants in the questionnaire.

Collected data were verified and coded prior to computerized data entry. The researcher utilized the Statistical Package for Social Sciences (SPSS version 20.0) for data entry and analysis. Frequency, percentage, arithmetic mean, range and SD were used as descriptive statistics. Healthcare workers' knowledge score regarding medical ethics was calculated as follow; the participated healthcare workers were asked to answer questions about the different aspects of medical ethics. Right answer is giving the score 1 while wrong answer of the answer of 'I don't know' were given the score zero. The overall score was calculated in the way that the higher the score, the higher the knowledge regarding medical ethics (the score ranged between 0-16).

Bivariate analyses of mean scores with regard to independent variables were done by ANOVA test for comparison of more than two groups. Least significance difference test (LSD) test was used for post hoc comparisons of ANOVA. Student's t-test was applied for comparison of the mean score of two different groups. Chisquare test was utilized for testing the association and/or difference between categorical variables. A p-value of less than 0.05 will be adopted for statistical significance.

Healthcare workers' knowledge was categorized according to the percentage of right answers into four categories; bad $(<60 \%)$, good $(60-<75 \%)$, very good $(75-<85 \%)$ and excellent $(\geq 85 \%)$.

Table 1: Baseline characteristics of the participants $(n=224)$.

\begin{tabular}{lcc}
\hline Characteristics & No. & $\%$ \\
\hline Age in years & \multicolumn{2}{c}{43.3} \\
$\leq 30$ & 97 & 33.5 \\
$31-40$ & 52 & 23.2 \\
$>40$ & \multicolumn{2}{c}{$24-52$} \\
Range (years) & $31.7 \pm 6.0$ \\
Mean \pm SD (years) & \multicolumn{2}{c}{} \\
Gender & 56 & 25.0 \\
Male & 168 & 75.0 \\
Female & \multicolumn{2}{c}{} \\
Nationality & 41 & 18.3 \\
Saudi & 183 & 81.7 \\
Non-Saudi & \multicolumn{2}{c}{} \\
Job & 74 & 33.0 \\
Doctor & 150 & 67.0 \\
Nurse & \multicolumn{2}{|c}{} \\
Duration of experience (years) & 108 & 48.2 \\
$\leq 5$ & 62 & 27.7 \\
6-10 & 54 & 24.1 \\
\hline 10 & & \\
\hline
\end{tabular}




\section{RESULTS}

Overall, 224 of 270 health care workers, invited to participate in the study by filling out the research questionnaire, returned completed questionnaires, giving a response rate of $83.0 \%$ (72.5\% for physicians (74/102) and $89.3 \%$ for nurses (150/168).

The study included 224 healthcare workers. Their baseline characteristics are presented in table (1). Their age ranged between 24 and 52 years with a mean of 31.7(SD 6.0) years. Three-quarters of them $(168 ; 75.0 \%)$ were females. Most of them were non-Saudi (183; 81.7\%). Almost two-thirds of them (150; $67.0 \%)$ were nurses and one-third $(74 ; 33.0 \%)$ were doctors. The experience was five years or less among almost half of them (108; $48.2 \%$ ) and more than ten years among 54 healthcare workers $(24.1 \%)$.

Table 2:- Mean of score reflecting knowledge of the healthcare workers about medical ethics (range 0-16) according their age.

\begin{tabular}{lccc}
\hline Age in years & Mean & SD & $\mathbf{P}^{*}$ \\
\hline $\mathbf{S 3 0}(\mathbf{9 7 )}$ & 10.32 & 2.16 & \\
$\mathbf{3 1 - 4 0}(\mathbf{7 5 )}$ & 9.53 & 2.77 & \\
$\mathbf{> 4 0}(\mathbf{5 2})$ & 9.63 & 3.15 & 0.109 \\
\hline
\end{tabular}

${ }^{*}$ ANOVA test

Table 3:- Mean of score reflecting knowledge of the healthcare workers about medical ethics (range 0-16) according their gender.

\begin{tabular}{lccc}
\hline Gender & Mean & SD & $\mathbf{P}^{*}$ \\
\hline Males (56) & 9.82 & 2.80 & 0.804 \\
Females (168) & 9.92 & 2.59 & \\
\hline
\end{tabular}

${ }^{*}$ Student's t-test

Table 4:- Mean of score reflecting knowledge of the healthcare workers about medical ethics (range 0-16) according their job.

\begin{tabular}{lccc}
\hline Job & Mean & SD & $\mathbf{P}^{*}$ \\
\hline Doctor (74) & 9.32 & 2.94 & 0.022 \\
Nurse (150) & 10.18 & 2.44 & \\
\hline
\end{tabular}

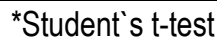

The majority of healthcare workers knew correctly that every patient must be treated with honesty and dignity $(214 ; 95.5 \%)$, in case of illegal pregnancy abortion cannot be recommended (195; $87.1 \%$ ) and patients with high socioeconomics class should not be treated with special care $(187 ; 83.5 \%)$. Most of health care workers knew correctly that confidentiality can be kept in modern care and should be abandoned (179; 79.9\%), patient should always be told if something is wrong $(173 ; 77.2 \%)$ and children should never be treated without the consent of their parents or guardians (except in an emergency) (166; 74.1\%). Almost twothirds of health care workers know correctly that patients not only need to consent for operations but also for tests or medications $(155 ; 69.2 \%)$, doctors and nurses should not refuse to treat patients who behave violently $(150 ; 67 \%)$ and ethical conduct is not important only to avoid legal action (149; 66.5\%). Only almost one-third of health care workers or less recognized correctly that in case of fetal malformation abortion cannot be offered (77; $34.4 \%)$, close relatives must not always be told about a patient's condition $(73 ; 32.6 \%)$ and only 41 health care workers (18.3\%) answered correctly that patient not should be informed immediately if he has incurable disease.

As obvious from figure 1, $42.4 \%$ of healthcare workers had bad knowledge regarding medical ethics, mostly doctors $(56.8 \%$ versus $35.3 \%$ of nurses). Very good or excellent level of medical ethics knowledge was reported among $21.4 \%$ and $7.1 \%$ of healthcare workers, respectively. Nurses reported more significant very good and excellent knowledge than doctors $(30 \%$ versus $25.7 \%)$.

The mean score reflecting knowledge of healthcare workers about medical ethics was highest among healthcare workers aged 30 years or less (10.32 \pm 2.16$)$ and lowest among those aged 31-40 years $(9.53 \pm 2.77)$. However, this difference was not statistically significant, $p=0.109$. Table 2

The mean score reflecting knowledge of healthcare workers about medical ethics was slightly higher among female healthcare workers than males. This difference was not statistically significant, $p=0.804$. Table 3

The mean score reflecting knowledge of healthcare workers about medical ethics was significantly higher among nurses than doctors (10.18 \pm 2.44 versus $9.32 \pm 2.94), p=0.022$. Table 4

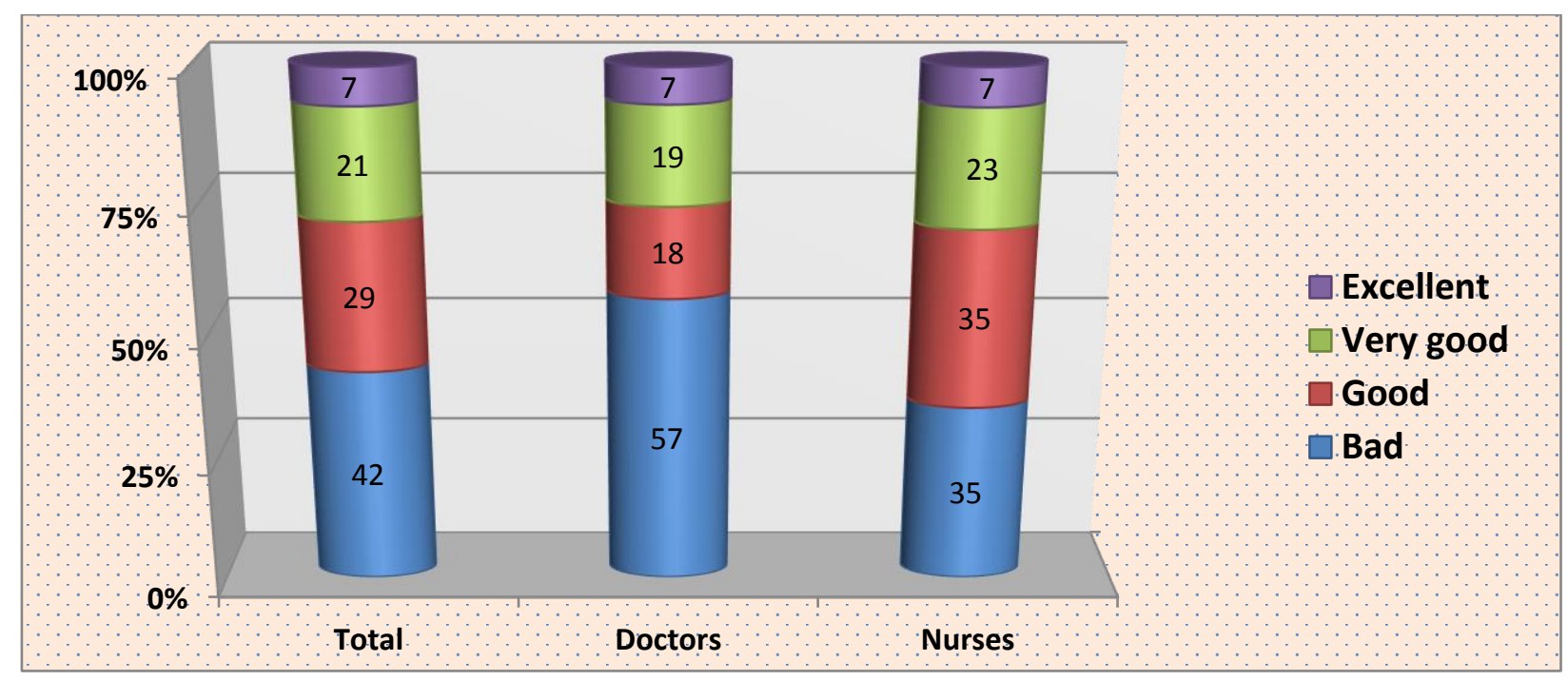

Fig 1: Knowledge of medical ethics among healthcare workers, PMMH 
Table 5:- Mean of score reflecting knowledge of the healthcare workers about medical ethics (range 0-16) according their nationality.

\begin{tabular}{lccc}
\hline Nationality & Mean & SD & P $^{*}$ \\
\hline Saudi (41) & 9.71 & 2.84 & 0.611 \\
Non-Saudi (183) & 9.94 & 2.60 & \\
\hline
\end{tabular}

*Student's t-test

Table 6:- Mean of score reflecting knowledge of the healthcare workers about medical ethics (range 0-16) according their experience.

\begin{tabular}{lccc}
\hline Experience in years & Mean & SD & $\mathbf{P}^{*}$ \\
\hline $\mathbf{S 5}(\mathbf{1 0 8 )}$ & 10.03 & 2.35 & \\
$\mathbf{6 - 1 0}(\mathbf{6 2})$ & 9.45 & 2.84 & \\
$\mathbf{> 1 0}$ (54) & 10.15 & 2.92 & 0.285 \\
\hline
\end{tabular}

${ }^{*}$ ANOVA test

The mean score reflecting knowledge of Saudi healthcare workers about medical ethics was not significantly different from that of non-Saudi healthcare workers, $\mathrm{p}=0.611$. Table 5

The mean score reflecting knowledge of healthcare workers about medical ethics was not significantly associated with their experience, $p=0.285$. Table 6

\section{DISCUSSION}

The study included 224 healthcare workers with a response rate of $83 \%$. This relatively high response rate, compared to that has been reported by Roberts, et al in USA, $2005(58 \%)^{4}$ and can probably be ascribed to the personal contact with the residents (both face to face and through e-mail communication) as well as to the explanation of the purpose of the study, scientific importance and value of the study to each resident. According to Rosnow and Rosenthal (1999) 5 these techniques (e.g. personal contact, using reminders and explaining the scientific importance and value of the study, ensuring the participants confidentiality) are linked to increase participation in surveys.

Up to our knowledge, this type of survey was the first of its kind in Taif, Saudi Arabia and of importance because the findings raise some fundamental and important issues for ethics education. The varying opinions of the healthcare personnel with respect to the day-to-day ethical issues, point to the need for appropriate training among healthcare staff and sensitize them to these issues in the workplace.

There was an obvious support for the informed consent. This may be due to the trend of increasing litigation against healthcare personnel by patients. However, the responses to the question regarding confidentiality were quite varying. While most of the participants agreed to the view that patient confidentiality is very important and should not be abandoned, only one-third of the respondents also felt that patient's relatives should not be told of the patient's condition. This may be perhaps due to the impact of the communitarian concept which is quite expected in Saudi culture. The cross-cultural dimension and difference in patient approach has been one of the major issues in present day bioethics. ${ }^{6}$

Continuing treatment for a non-compliant patient has always been an ethical dilemma. This situation is largely influenced by the system of healthcare delivery and in managed care settings it is difficult to both continue and abandon the management of such a patient. ${ }^{7}$ In the present study, the opinion against discontinuing the management of a patient when the patient refuses to undergo treatment may reflect the influence of the socio-cultural background of the respondents. Doctors tended more to refuse treatment of non-compliant patients than nurses. The opinion against discontinuing the management of a patient if he is violent again may reflect the influence of the socio-cultural background of the respondents. However it may also reflect the supererogatory view of the respondents.

The four principles of ethics based on the western thoughts namely autonomy, justice, beneficence and non-maleficence, may not be a globally applicable framework. ${ }^{8}$ Even in western countries such as the United Kingdom there are dissenting voices regarding the application of the principle of autonomy in medical field which are being supported by an argument that it erodes the trust between the patient and the treating physician. ${ }^{9}$ Hence it is not surprising that around $40 \%$ of the respondents in the present study did not agree to the view that the patient's wishes should always be adhered to. A percentage of $45 \%$ had been reported in another study conducted in Caribbean region. ${ }^{3}$

Volumes have been written about abortion, although the crosscultural dimensions in practicing abortion are missed in the western literature. ${ }^{10}$ In the present study, the overwhelming disagreement for abortion may reflect the influence of religious values in the respondents. In Saudi Arabia, efforts to legalize abortion in certain circumstances have been recently discussed among Senior Religious Scholars and specialized physicians to permit abortions in certain circumstances. The Council of Senior Scholars issued a legal opinion (Fatwa-240) on this subject on 16 January 2011, and based on the two verses from the Holy Quran: (But whoever is forced [by necessity], neither desiring [it] nor transgressing [its limit], there is no sin upon him. Indeed, Allah is Forgiving and Merciful). (Al-Baqara-173) and (He has chosen you and has not placed upon you in the religion any difficulty) (Al-Haj78), The legal opinion came up with the following items: It is permissible to abort a malformed foetus after 120 days of conception (19 weeks of gestation) (when the soul joins its body) if the continuation of pregnancy is expected to result in the death of its mother. It is permissible to abort a malformed foetus before 120 days of conception, if its death is expected following delivery, or if the foetus has severe disabilities that cannot be cured. The foetus can be aborted at any stage of pregnancy, if its death is medically confirmed in the womb of its mother. In all circumstance, it is not permissible to abort a foetus without a medical report from a specialized and trustworthy committee that is composed of at least three physicians, after obtaining a written consent from parents or the mother alone if the continuation of pregnancy is affecting her health. The consent can be obtained by the delegates of parents, if they cannot give it for any reason. The signed consent must be kept in the medical record of the mother. ${ }^{11}$

The strengths of this study are its focus on salient practical ethics and ethically important professional development issues. In addition, the strengths of this study include the solid response rate, the comprehensiveness of the survey. Nevertheless, this survey study has several limitations. It relies on self-report, and it involved a sample at a single institution. The response rate of $83 \%$ 
was high, but does not prevent sampling bias. However, the concordance of our findings with similar work and the inclusion of a relatively large sample size, however, suggest that the results may be generalizable.12-15 The cross-sectional design provides insights into developmental issues relevant to professionalism and ethics education in medicine but does not show changes as training progresses as permitted by a longitudinal design.

Family physicians are often in the front line of organizational and social change and must be able to adapt to the increasing complexity of medical care options, expectations of personal and professional accountability, the predicted increase in cultural diversity of patients, and the constant evolution in health system organization. ${ }^{16}$ There is also an expectation that medical ethics training will equip the family physician for a future role as manager of health care resources, advocate for patients and communities, and leader for organizational change. Training in medical ethics is recognized as fundamentally important to the practice of family medicine by professional organizations, accreditation agencies, and by society. ${ }^{16}$ In the present study, level of knowledge among nurses was significantly higher than physicians.

Among limitations of the study, it includes healthcare workers in one setting in Taif, Saudi Arabia, and the results may not be representative of the whole nation. In addition, the study findings merely convey associations rather than inferences because of the study design adopted.

Conclusively, a considerable proportion of healthcare workers in $\mathrm{PMMH}$ had bad knowledge regarding medical ethics, mostly doctors. Nurses reported more significant medical ethics knowledge than doctors.

\section{REFERENCES}

1. Gatrad AR, Sheikh A. Medical ethics and Islam: principles and practice. Arch. Dis. Child. 2001; 84; 72-75.

2. Elsayed DM, Ahmed RM. Medical Ethics: What is it? Why is it important? Sudanese Journal of public health April 2009; 4(2):126-133

3. Hariharan A, Jonnalagadda R, Gora J. Knowledge, attitudes and practices of healthcare personnel towards Care-Ethics: $A$ perspective from the Caribbean, The Internet Journal of Law, Healthcare and Ethics 2007; 5(1):

4. Roberts LW, Geppert CM, Warner TD, Hammond KA, Lamberton LP. Bioethics principles, informed consent, and ethical care for special populations: curricular needs expressed by men and women physicians in training. Psychosomatics 2005; 46(5): 440-450.

5. Rosnow R, Rosenthal R. Psychology; Social sciences; Research; Methodology In: Beginning behavioral research: A conceptual primer. Published by: Prentice Hall (Upper Saddle River, NJ), 3rd edition, 1999; 475-81.
6. Kuczewski, M and PJ McCruden 2001. Informed Consent: Does it take a Village? The Problem of Culture and Truth Telling. Cambridge Quarterly of Healthcare Ethics 10: 34-46

7. Browne A, Dickson B, van Der Wal R. The Ethical management of the Noncompliant Patient. Cambridge Quarterly of Healthcare Ethics 2003;12: 289-299

8. Takala, T 2001. What is Wrong with Global Bioethics? On the Limitations of the Four Principles Approach. Cambridge Quarterly of Healthcare Ethics 10: 72-77

9. Hariharan S, Moseley HSL, Kumar AY, Walrond ER, Ramesh J. Futility-of-care decisions in the treatment of moribund intensive care patients in a developing country. Canadian Journal of Anesthesia 2003;50: 847-852

10. Nie J-B. The Problem of coerced abortion in China. Cambridge Quarterly of Healthcare Ethics 1999; 8: 463-479

11. Al-Alaiyan S, AlFaleh KM. Aborting a malformed fetus: A debatable issue in Saudi Arabia. J Clin Neonatol 2012; 1:6-11

12. Olukoya AA: Attitudes of medical students to medical ethics in their curriculum. Med Educ 1983; 17:83-86

13. Jacobson JA, Tolle SW, Stocking C, Siegler M: Internal medicine residents' preferences regarding medical ethics education. Acad Med 1989; 64:760-764

14. American Association of Medical Colleges. Learning objectives for medical student education--guidelines for medical schools: report I of the Medical School Objectives Project. Acad Med. 1999 Jan; 74(1):13-8.

15. Howe KR. Medical students' evaluations of different levels of medical ethics teaching: implications for curricula. Med Educ 1987; 21:340-349

16. Manson $\mathrm{H}$. The Need for Medical Ethics Education in Family Medicine Training. Fam Med 2008; 40(9):658-64.

\section{Source of Support: Nil.}

\section{Conflict of Interest: None Declared.}

Copyright: (c) the author(s) and publisher. IJMRP is an official publication of Ibn Sina Academy of Medieval Medicine \& Sciences, registered in 2001 under Indian Trusts Act, 1882.

This is an open access article distributed under the terms of the Creative Commons Attribution Non-commercial License, which permits unrestricted non-commercial use, distribution, and reproduction in any medium, provided the original work is properly cited.

Cite this article as: Muied A. AL Qarny, Amal M. Alsofiani, Ali Githan AL Garny. Medical Ethics Knowledge and Practice among Healthcare Workers at Prince Mansour Military Hospital (PMMH), Taif, Saudi Arabia, 2012. Int J Med Res Prof. 2016; 2(4):127-31. 